\title{
PRECISE ASYMPTOTICS FOR THE MOMENT CONVERGENCE OF MOVING-AVERAGE PROCESS UNDER DEPENDENCE
}

\author{
Qing-Pei Zang And Ke-Ang Fu
}

\begin{abstract}
Let $\left\{\varepsilon_{i}:-\infty<i<\infty\right\}$ be a strictly stationary sequence of linearly positive quadrant dependent random variables and $\sum_{i=-\infty}^{\infty}\left|a_{i}\right|<$ $\infty$. In this paper, we prove the precise asymptotics in the law of iterated logarithm for the moment convergence of moving-average process of the form $X_{k}=\sum_{i=-\infty}^{\infty} a_{i+k} \varepsilon_{i}, k \geq 1$.
\end{abstract}

\section{Introduction}

We assume that $\left\{\varepsilon_{i}:-\infty<i<\infty\right\}$ is a doubly infinite sequence of identically distributed variables. Let $\left\{a_{i}:-\infty<i<\infty\right\}$ be an absolutely summable sequence of real numbers and $X_{k}=\sum_{i=-\infty}^{\infty} a_{i+k} \varepsilon_{i}, k \geq 1$. Set $S_{n}=\sum_{k=1}^{n} X_{k}$, also let $\log y=\log (y \vee e), \log \log y=\log \log \left(y \vee e^{e}\right)$ for all $y>0$.

When $\left\{\varepsilon_{i}:-\infty<i<\infty\right\}$ is a sequence of independent random variables, many limiting results have been obtained for moving-average process $\left\{X_{k}\right.$ : $k \geq 1\}$. For example, Burton and Dehling [1] have obtained a large deviation principle for $\left\{X_{k}: k \geq 1\right\}$ assuming $E \exp t \varepsilon_{1}<\infty$ for all $t$, Ibragimov [4] has established the central limit theorem for $\left\{X_{k}: k \geq 1\right\}$, Li et al. [7] derived convergence rates of moderate deviations and the precise asymptotics in the law of the iterated logarithm.

On the other hand, Gut and Spătaru [3] proved the precise asymptotics of i.i.d random variables. One of their results is as follows.

Theorem A. Suppose that $\left\{Y_{k}: k \geq 1\right\}$ is a sequence of i.i.d random variables with $E Y_{1}=0$ and $E Y_{1}^{2}=\sigma^{2}<\infty$. Then

$$
\lim _{\varepsilon \searrow 0} \varepsilon^{2} \sum_{n=1}^{\infty} \frac{1}{n \log n} P\left(\left|\sum_{k=1}^{n} Y_{k}\right| \geq \varepsilon \sqrt{n \log \log n}\right)=\sigma^{2} .
$$

Received December 15, 2008; Revised June 11, 2009.

2000 Mathematics Subject Classification. 60F99, 60G20.

Key words and phrases. precise asymptotics, moving-average, linear positive quadrant dependence. 
Chow [2] discussed the complete moment convergence of i.i.d random variables. He got the following result:

Theorem B. Let $\left\{Y, Y_{k}: k \geq 1\right\}$ be a sequence of i.i.d random variables with $E Y_{1}=0$. Suppose that $p \geq 1, \alpha>\frac{1}{2}, p \alpha>1, E\left\{|Y|^{p}+|Y| \log (1+|Y|)\right\}<\infty$. Then for any $\varepsilon>0$, we have

$$
\sum_{n=1}^{\infty} n^{p \alpha-2-\alpha} E\left\{\max _{j \leq n}\left|\sum_{k=1}^{j} Y_{k}\right|-\varepsilon n^{\alpha}\right\}_{+}<\infty
$$

In this note, we show that the precise asymptotics for the moment convergence holds for moving-average process when $\left\{\varepsilon_{i}:-\infty<i<\infty\right\}$ is a strictly stationary linear positive quadrant dependent sequence. First, we shall give the definition of linear positive quadrant dependent sequence.

Two random variables $X$ and $Y$ are said to be positive quadrant dependent (PQD) if $P(X>x, Y>y) \geq P(X>x) P(Y>y)$ for all $x, y \in R$. This notation was first introduced by Lehmann [6], another concept which is stronger than PQD was due to Newman [9]: a sequence $\left\{\varepsilon_{i}:-\infty<i<\infty\right\}$ is said to be linear positive quadrant dependent (LPQD) if for any disjoint finite subsets $A, B \subset\{\ldots,-2,-1,0,1,2, \ldots\}$ and any positive real numbers $r_{j}$,

$$
\sum_{i \in A} r_{i} \varepsilon_{i} \text { and } \sum_{j \in B} r_{j} \varepsilon_{j} \text { are } P Q D \text {. }
$$

\section{Main result}

Throughout this paper, let $\left\{\varepsilon_{i}:-\infty<i<\infty\right\}$ be a sequence of strictly stationary linear positive quadrant dependent random variables with $E \varepsilon_{i}=0$, $0<E \varepsilon_{i}^{2}<\infty$, and set $0<\sigma^{2}=E \varepsilon_{1}^{2}+2 \sum_{k=2}^{\infty} E \varepsilon_{1} \varepsilon_{k}<\infty$ unless it is specially mentioned. Now we state our result as follows.

Theorem 2.1. Assume

$$
\sum_{i=n+1}^{\infty} E \varepsilon_{1} \varepsilon_{i}=O\left(n^{-\rho}\right) \text { for some } \rho>0,
$$

and

$$
E\left|\varepsilon_{i}\right|^{s}<\infty \text { for some } s>2 .
$$

Then for $-1<b<-1 / 2$, we have

$\lim _{\varepsilon \searrow 0} \varepsilon^{2(b+1)} \sum_{n=1}^{\infty} \frac{(\log \log n)^{b}}{n^{\frac{3}{2}} \log n} E\left\{\left|S_{n}\right|-\varepsilon \sqrt{2 n \log \log n}\right\}_{+}=\frac{2^{-b-1}}{(b+1)(2 b+3)} E|Z|^{2 b+3}$,

where $Z$ has a normal distribution with mean 0 and variance $\tau^{2}=\sigma^{2}\left(\sum_{i=-\infty}^{\infty} a_{i}\right)^{2}$.

Remark 2.1. Let $a_{i+k}=1, i=k ; a_{i+k}=0, i \neq k, 1 \leq k \leq n$. Then $X_{k}=$ $\varepsilon_{k}, S_{n}=\sum_{k=1}^{n} \varepsilon_{k}$. Thus above result holds under some suitable conditions when $\left\{X_{i}: i \geq 1\right\}$ is a sequence of strictly stationary linear positive quadrant dependent random variables. 
The following example comes from Li and Wang [8].

Remark 2.2. A finite family of random variables $\left\{X_{i}: 1 \leq i \leq n\right\}$ is said to be positively associated (PA) if for every pair of disjoint subsets $A$ and $B$ of $\{1,2, \ldots\}$,

$$
\operatorname{Cov}\left\{f\left(X_{i}: i \in A\right), g\left(X_{j} ; j \in B\right)\right\} \geq 0
$$

whenever $f$ and $g$ are coordinatewise increasing and the covariance exists. A PA sequence is obviously a LPQD sequence, the following example shows that LPQD does not imply PA: Consider three discrete random variables with joint density $p(x, y, z):=P(X=x, Y=y, Z=z)$.

$$
\begin{aligned}
p(2,2,1) & =p(3,2,1)=p(2,3,1)=p(3,3,1)=p(1,1,2) \\
& =p(2,1,2)=p(3,1,2)=p(1,2,2)=p(1,3,2)=\frac{1}{17} \quad \text { and } \\
p(1,1,1) & =p(3,3,2)=\frac{4}{17} .
\end{aligned}
$$

A lengthy verification shows that $\{X, Y, Z\}$ is LPQD. But, $\{X, Y, Z\}$ is not PA since $P(X>1, Y>1, Z>1)=\frac{4}{17}<P(X>1, Y>1) P(Z>1)=\frac{72}{289}$.

\section{Some lemmas}

First, we give some lemmas which will be used in the proofs. Lemma 3.1 and Lemma 3.2 are from Burton and Dehling [1], Kim [5] respectively.

Lemma 3.1. Let $\sum_{i=-\infty}^{\infty} a_{i}$ be an absolutely convergent series of real numbers with $a=\sum_{i=-\infty}^{\infty} a_{i}$ and $k \geq 1$. Then

$$
\lim _{n \rightarrow \infty} \frac{1}{n} \sum_{i=-\infty}^{\infty}\left|\sum_{j=i+1}^{i+n} a_{j}\right|^{k}=|a|^{k} .
$$

Lemma 3.2. Let $\left\{\varepsilon_{i}:-\infty<i<\infty\right\}$ be a sequence of strictly stationary linear positive quadrant dependent random variables with $E \varepsilon_{i}=0,0<E \varepsilon_{i}^{2}<\infty$, and set $0<\sigma^{2}=E \varepsilon_{1}^{2}+2 \sum_{k=2}^{\infty} E \varepsilon_{1} \varepsilon_{k}<\infty$. Assume

$$
\sum_{i=n+1}^{\infty} E \varepsilon_{1} \varepsilon_{i}=O\left(n^{-\rho}\right) \text { for some } \rho>0
$$

and

$$
E\left|\varepsilon_{i}\right|^{s}<\infty \text { for some } s>2 \text {. }
$$

Then the linear process $\left\{X_{k}\right\}$ fulfills the CLT, that is,

$$
\frac{S_{n}}{\tau \sqrt{n}} \stackrel{\mathcal{D}}{\longrightarrow} N(0,1), \text { where } \tau=\sigma \sum_{i=-\infty}^{\infty} a_{i} .
$$

Throughout the sequel, $N$ represent standard normal variable. $C$ will denote a positive constant although its value may change from one appearance to the next and let $[x]$ indicate the maximum integer not larger than $x$. 


\section{Proof of Theorem 2.1}

Without loss of generality, we assume $\tau=1$ in this section. Let $A(\varepsilon)=$ $\exp \left\{\exp \left\{\frac{M}{\varepsilon^{2}}\right\}\right\}, M>1$. Our main result will be proved via the following propositions.

Proposition 4.1. For any $b>-1$, we have

$\lim _{\varepsilon \searrow 0} \varepsilon^{2(b+1)} \sum_{n=1}^{\infty} \frac{(\log \log n)^{b}}{n \log n} E\{|N|-\varepsilon \sqrt{2 \log \log n}\}_{+}=\frac{2^{-b-1}}{(b+1)(2 b+3)} E|N|^{2 b+3}$.

Proof. By the variable change, we have

$$
\begin{aligned}
& \lim _{\varepsilon \searrow 0} \varepsilon^{2(b+1)} \sum_{n=1}^{\infty} \frac{(\log \log n)^{b}}{n \log n} E\{|N|-\varepsilon \sqrt{2 \log \log n}\}_{+} \\
= & \lim _{\varepsilon \searrow 0} \varepsilon^{2(b+1)} \sum_{n=1}^{\infty} \frac{(\log \log n)^{b}}{n \log n} \int_{\varepsilon \sqrt{2 \log \log n}}^{\infty} P(|N| \geq x) d x \\
= & \lim _{\varepsilon \searrow 0} \varepsilon^{2(b+1)} \int_{e^{e}}^{\infty} \frac{(\log \log t)^{b}}{t \log t} \int_{\varepsilon \sqrt{2 \log \log t}}^{\infty} P(|N| \geq x) d x d t \\
= & \lim _{\varepsilon \searrow 0} 2^{-b} \int_{\varepsilon \sqrt{2}}^{\infty} y^{2 b+1} \int_{y}^{\infty} P(|N| \geq x) d x d y \\
= & \lim _{\varepsilon \searrow 0} \frac{2^{-b}}{2(b+1)} \int_{\varepsilon \sqrt{2}}^{\infty} P(|N| \geq x)\left(x^{2 b+2}-\varepsilon^{2 b+2} \cdot 2^{b+1}\right) d x \\
= & \lim _{\varepsilon \searrow 0} \frac{2^{-b}}{2(b+1)} \int_{\varepsilon \sqrt{2}}^{\infty} x^{2 b+2} P(|N| \geq x) d x \\
= & \frac{2^{-b-1}}{(b+1)(2 b+3)} E|N|^{2 b+3} .
\end{aligned}
$$

Thus the proposition is now proved.

Proposition 4.2. For any $b>-1$, we have

$\lim _{\varepsilon \searrow 0} \varepsilon^{2(b+1)} \sum_{n \leq A(\varepsilon)} \frac{(\log \log n)^{b}}{n^{\frac{3}{2}} \log n}\left|E\left\{\left|S_{n}\right|-\varepsilon \sqrt{2 n \log \log n}\right\}_{+}-\sqrt{n} E\{|N|-\varepsilon \sqrt{2 \log \log n}\}_{+}\right|=0$.

Proof. Denote

$$
\triangle_{n}=\sup _{x}\left|P\left(\frac{\left|S_{n}\right|}{\sqrt{n}} \geq x\right)-P(|N| \geq x)\right|,
$$

it follows from Lemma 3.2 that $\triangle_{n} \rightarrow 0$ as $n \rightarrow \infty$. Then

$$
\sum_{n \leq A(\varepsilon)} \frac{(\log \log n)^{b}}{n^{\frac{3}{2}} \log n}\left|E\left\{\left|S_{n}\right|-\varepsilon \sqrt{2 n \log \log n}\right\}_{+}-\sqrt{n} E\{|N|-\varepsilon \sqrt{2 \log \log n}\}_{+}\right|
$$


$\leq \sum_{n \leq A(\varepsilon)} \frac{(\log \log n)^{b}}{n \log n} \times \int_{0}^{\infty}\left|P\left(\frac{\left|S_{n}\right|}{\sqrt{n}} \geq \varepsilon \sqrt{2 \log \log n}+x\right)-P(|N| \geq \varepsilon \sqrt{2 \log \log n}+x)\right| d x$

$\leq \sum_{n \leq A(\varepsilon)} \frac{(\log \log n)^{b}}{n \log n}\left(\triangle_{n_{1}}+\triangle_{n_{2}}\right)($ say $)$,

where

$$
\begin{aligned}
& \triangle_{n_{1}}=\int_{0}^{\frac{1}{\sqrt{\triangle_{n}}}}\left|P\left(\frac{\left|S_{n}\right|}{\sqrt{n}} \geq \varepsilon \sqrt{2 \log \log n}+x\right)-P(|N| \geq \varepsilon \sqrt{2 \log \log n}+x)\right| d x, \\
& \triangle_{n_{2}}=\int_{\frac{1}{\sqrt{\Delta_{n}}}}^{\infty}\left|P\left(\frac{\left|S_{n}\right|}{\sqrt{n}} \geq \varepsilon \sqrt{2 \log \log n}+x\right)-P(|N| \geq \varepsilon \sqrt{2 \log \log n}+x)\right| d x .
\end{aligned}
$$

It is easy to obtain

$$
\triangle_{n_{1}} \leq \sqrt{\triangle_{n}} \rightarrow 0 \text { as } n \rightarrow \infty
$$

Next, observe that

$$
\sum_{k=1}^{n} X_{k}=\sum_{i=-\infty}^{\infty} \sum_{k=1}^{n} a_{k+i} \varepsilon_{i} .
$$

Set $a_{n i}=\sum_{k=1}^{n} a_{k+i}$. Then

$$
\sum_{k=1}^{n} X_{k}=\sum_{i=-\infty}^{\infty} a_{n i} \varepsilon_{i}=\sum_{i=-\infty}^{\infty} Y_{i} \text { (say). }
$$

From Lemma 3.1, we can assume, without loss of generality, that

$$
\sum_{i=-\infty}^{\infty}\left|a_{n i}\right| \leq n, n \geq 1 \text { and } \sum_{i=-\infty}^{\infty}\left|a_{i}\right| \leq 1 .
$$

And then, by Lemma 3.1 and the stationarity we get

$$
\begin{aligned}
\operatorname{Var}\left(S_{n}\right) & =E \varepsilon_{1}^{2} \sum_{i=-\infty}^{\infty} a_{n i}^{2}+2 \sum_{i=-\infty}^{\infty} \sum_{j=i+1}^{\infty} a_{n i} a_{n j} E \varepsilon_{i} \varepsilon_{j} \\
& \leq n C E \varepsilon_{1}^{2}+2 \sum_{i=-\infty}^{\infty} \sum_{k=1}^{\infty} a_{n i} a_{n k+i} E \varepsilon_{1} \varepsilon_{k+1} \\
& \leq n C E \varepsilon_{1}^{2}+\sum_{i=-\infty}^{\infty} \sum_{k=1}^{\infty}\left(a_{n i}^{2}+a_{n k+i}^{2}\right) E \varepsilon_{1} \varepsilon_{k+1} \\
& \leq n C E \varepsilon_{1}^{2}+\sum_{k=1}^{\infty} E \varepsilon_{1} \varepsilon_{k+1} \sum_{i=-\infty}^{\infty} a_{n i}^{2}+\sum_{k=1}^{\infty} E \varepsilon_{1} \varepsilon_{k+1} \sum_{i=-\infty}^{\infty} a_{n k+i}^{2} \\
& \leq C n .
\end{aligned}
$$

Thus, by virtues of Markov's inequality, we have

$$
\triangle_{n_{2}} \leq \int_{\frac{1}{\sqrt{\triangle_{n}}}}^{\infty} \frac{C+1}{(\varepsilon \sqrt{\log \log n}+x)^{2}} d x \leq(C+1) \sqrt{\triangle_{n}}
$$


Denote $\triangle_{n}^{\prime}=\triangle_{n_{1}}+\triangle_{n_{2}}$. It follows that

$$
\frac{1}{(\log \log m)^{b+1}} \sum_{n=1}^{m} \frac{\triangle_{n}^{\prime}(\log \log n)^{b}}{n \log n} \rightarrow 0 \text { as } m \rightarrow \infty
$$

We have

$$
\begin{aligned}
& \lim _{\varepsilon \searrow 0} \varepsilon^{2(b+1)} \sum_{n \leq A(\varepsilon)} \frac{(\log \log n)^{b}}{n^{\frac{3}{2}} \log n}\left|E\left\{\left|S_{n}\right|-\varepsilon \sqrt{2 n \log \log n}\right\}_{+}-\sqrt{n} E\{|N|-\varepsilon \sqrt{2 \log \log n}\}_{+}\right| \\
\leq & \lim _{\varepsilon \searrow 0} \varepsilon^{2(b+1)} \sum_{n \leq A(\varepsilon)} \frac{(\log \log n)^{b}}{n \log n} \triangle_{n}^{\prime} \\
= & \lim _{\varepsilon \searrow 0} M^{b+1} \frac{1}{(\log \log [A(\varepsilon)])^{b+1}} \sum_{n \leq A(\varepsilon)} \frac{\triangle_{n}^{\prime}}{n \log n}(\log \log n)^{b} \rightarrow 0 .
\end{aligned}
$$

Hence, the proposition holds.

Proposition 4.3. Uniformly for $0<\varepsilon<\frac{1}{\sqrt{2}}$, we have

$$
\lim _{M \longrightarrow \infty} \limsup _{\varepsilon \backslash 0} \varepsilon^{2(b+1)} \sum_{n>A(\varepsilon)} \frac{(\log \log n)^{b}}{n^{\frac{3}{2}} \log n}\left|E\left\{\left|S_{n}\right|-\varepsilon \sqrt{2 n \log \log n}\right\}_{+}-\sqrt{n} E\{|N|-\varepsilon \sqrt{2 \log \log n}\}_{+}\right|=0 .
$$

Proof. It is sufficient to show

$$
\lim _{M \longrightarrow \infty} \varepsilon^{2(b+1)} \sum_{n>A(\varepsilon)} \frac{(\log \log n)^{b}}{n \log n} E\{|N|-\varepsilon \sqrt{2 \log \log n}\}_{+} \mid=0
$$

uniformly with respect to all sufficient small $0<\varepsilon<\frac{1}{\sqrt{2}}$, and

(1.3) $\lim _{M \longrightarrow \infty} \limsup _{\varepsilon \searrow 0} \varepsilon^{2(b+1)} \sum_{n>A(\varepsilon)} \frac{(\log \log n)^{b}}{n^{\frac{3}{2}} \log n} E\left\{\left|S_{n}\right|-\varepsilon \sqrt{2 n \log \log n}\right\}_{+}=0$.

Note that $A(\varepsilon)-1 \geq \sqrt{A(\varepsilon)}$ for $M>1$ and $0<\varepsilon<\frac{1}{\sqrt{2}}$. Thus

$$
\begin{aligned}
& \varepsilon^{2(b+1)} \sum_{n>A(\varepsilon)} \frac{(\log \log n)^{b}}{n \log n} E\{|N|-\varepsilon \sqrt{2 \log \log n}\}_{+} \\
\leq & \varepsilon^{2(b+1)} \int_{A(\varepsilon)-1}^{\infty} \frac{(\log \log y)^{b}}{y \log y} \int_{\varepsilon \sqrt{\log \log y}}^{\infty} P\{|N| \geq x\} d x d y \\
\leq & \varepsilon^{2(b+1)} \int_{\sqrt{A(\varepsilon)}}^{\infty} \frac{(\log \log y)^{b}}{y \log y} \int_{\varepsilon \sqrt{\log \log y}}^{\infty} P\{|N| \geq x\} d x d y
\end{aligned}
$$




$$
\begin{aligned}
& =2 \int_{\sqrt{M-\varepsilon^{2} \log 2}}^{\infty} t^{2 b+1} \int_{t}^{\infty} P\{|N| \geq x\} d x d t \\
& \leq 2 \int_{\sqrt{M-\frac{1}{2} \log 2}}^{\infty} t^{2 b+1} \int_{t}^{\infty} P\{|N| \geq x\} d t d x \\
& \leq 2 \int_{\sqrt{M-\frac{1}{2} \log 2}}^{\infty} P\{|N| \geq x\} \int_{\sqrt{M-\frac{1}{2} \log 2}}^{x} t^{2 b+1} d t d x \\
& \leq C \int_{\sqrt{M-\frac{1}{2} \log 2}}^{\infty} x^{2 b+2} P\{|N| \geq x\} d x \longrightarrow 0 \text { as } M \rightarrow \infty .
\end{aligned}
$$

Then (1.2) is proved.

Now we turn to prove (1.3). Notice that $E \varepsilon_{1}^{2}<\infty$, which coupled with (1.1), it follows that, for $-1<b<-1 / 2$

$$
\begin{aligned}
& \lim _{M \longrightarrow \infty} \limsup _{\varepsilon \searrow 0} \varepsilon^{2(b+1)} \sum_{n>A(\varepsilon)} \frac{(\log \log n)^{b}}{n^{\frac{3}{2}} \log n} E\left\{\left|S_{n}\right|-\varepsilon \sqrt{2 n \log \log n}\right\}_{+} \\
= & \lim _{M \longrightarrow \infty} \limsup _{\varepsilon \searrow 0} \varepsilon^{2(b+1)} \sum_{n>A(\varepsilon)} \frac{(\log \log n)^{b}}{n^{\frac{3}{2}} \log n} \int_{\varepsilon \sqrt{2 n \log \log n}}^{\infty} P\left(\left|\sum_{i=-\infty}^{\infty} a_{n i} \varepsilon_{i}\right| \geq x\right) d x \\
\leq & \lim _{M \longrightarrow \infty} \limsup _{\varepsilon \searrow 0} \varepsilon^{2(b+1)} \sum_{n>A(\varepsilon)} \frac{(\log \log n)^{b}}{n^{\frac{3}{2}} \log n} \int_{\varepsilon \sqrt{2 n \log \log n}}^{\infty} \frac{C n}{x^{2}} d x \\
\leq & \lim _{M \longrightarrow \infty} \limsup _{\varepsilon \searrow 0} \varepsilon^{2(b+1)} \sum_{n>A(\varepsilon)} \frac{(\log \log n)^{b}}{n^{\frac{1}{2}} \log n}(\varepsilon \sqrt{2 n \log \log n})^{-1} \\
\leq & \lim _{M \longrightarrow \infty} \limsup _{\varepsilon \searrow 0} \varepsilon^{2 b+1}[\log \log A(\varepsilon)]^{b+\frac{1}{2}} \\
\leq & \lim _{M \longrightarrow \infty} M^{b+\frac{1}{2}}=0 .
\end{aligned}
$$

Then, we complete the proof of this proposition.

Our main result now follows from the propositions.

Acknowledgement. The authors wish to express their deep gratitude to an anonymous referee for his(her) valuable comments on an earlier version which improve the quality of this paper.

\section{References}

[1] R. M. Burton and H. Dehling, Large deviations for some weakly dependent random processes, Statist. Probab. Lett. 9 (1990), no. 5, 397-401.

[2] Y. S. Chow, On the rate of moment convergence of sample sums and extremes, Bull. Inst. Math. Acad. Sinica 16 (1988), no. 3, 177-201.

[3] A. Gut and A. Spătaru, Precise asymptotics in the law of the iterated logarithm, Ann. Probab. 28 (2000), no. 4, 1870-1883.

[4] I. A. Ibragimov, Some limit theorems for stationary processes, Teor. Verojatnost. i Primenen. 7 (1962), 361-392. 
[5] T. S. Kim and J. I. Baek, A central limit theorem for stationary linear processes generated by linearly positively quadrant-dependent process, Statist. Probab. Lett. 51 (2001), no. 3, 299-305.

6] E. L. Lehmann, Some concepts of dependence, Ann. Math. Statist. 37 (1966), 1137-1153.

[7] D. L. Li, M. B. Rao, and X. C. Wang, Complete convergence of moving average processes, Statist. Probab. Lett. 14 (1992), no. 2, 111-114.

[8] Y. X. Li and J. F. Wang, The law of the iterated logarithm for positively dependent random variables, J. Math. Anal. Appl. 339 (2008), no. 1, 259-265.

[9] C. M. Newman, Asymptotic independence and limit theorems for positively and negatively dependent random variables, Inequalities in statistics and probability (Lincoln, Neb., 1982), 127-140, IMS Lecture Notes Monogr. Ser., 5, Inst. Math. Statist., Hayward, CA, 1984 .

QING-Pei Zang

FACULTY OF SCIENCE

JiANGSU UNIVERSITY

Zhenjiang 212013 P. R. China

AND

School of Mathematical Science

Huaiyin Normal University

Huaian 223300, P. R. China

E-mail address: zqphunhu@yahoo.com.cn

Ke-Ang Fu

School of Statistics and Mathematics

ZheJiAng Gongshang University

Hangzhou 310018, P. R. China

E-mail address: fukeang@126.com 